\title{
An Order-Preserving Linear Map from Matrices to Banach $*$-Algebras and Applications
}

\author{
Pattrawut Chansangiam \\ Department of Mathematics, Faculty of Science, King Mongkut's Institute of Technology Ladkrabang, Bangkok 10520, Thailand \\ Correspondence should be addressed to Pattrawut Chansangiam; kcpattra@kmitl.ac.th
}

Received 30 August 2012; Accepted 17 October 2012

Academic Editor: Tepper L. Gill

Copyright (C) 2013 Pattrawut Chansangiam. This is an open access article distributed under the Creative Commons Attribution License, which permits unrestricted use, distribution, and reproduction in any medium, provided the original work is properly cited.

We establish a theorem for which a number of absolute-value identities and inequalities in the framework of Banach $*$-algebras can be generated. To do this job, we construct an order-preserving linear map from the vector space of 2-by-2 hermitian matrices to a hermitian Banach $*$-algebras. This map can convert any suitable matrix ordering to a number of identities and inequalities in Banach *-algebras. Hence, we obtain a number of analogues of the well-known results in a framework of hermitian Banach *-algebras.

\section{Introduction}

There are many works involving absolute-value identities and inequalities. The famous such identities are the parallelogram law and the polarization identity. It is well known that these identities hold in the context of Euclidean spaces, normed linear spaces, and inner product spaces. On the other hand for inequalities, Bohr [1] established the classical Bohr's inequality which asserts that

$$
|a+b|^{2} \leqslant p|a|^{2}+q|b|^{2}
$$

for complex numbers $a, b$ and real numbers $p, q>1$ such that $1 / p+1 / q=1$. The equality in (1) occurs if and only if $p a=q b$. Then a number of extensions and variations of absolute-value inequalities concerning Bohr's inequality were developed in various contexts by many authors. The results for complex numbers are obtained in [2-4]. The context of matrices is given in [5]. Bohr's inequality and related results were generalized to operator algebras in [6-15].

The first version of operator Bohr's inequality was established via direct computations by Hirzallah [12]. Later, Hirzallah's results were extended by the same technique in [6, 10, 14]. Zhang [15] used operator identities and inequalities for approaching operator inequalities related to (1). Recently, the idea of matrix ordering for discussing operator absolutevalue inequalities appears in $[8,9,11]$. However, the results mentioned above has been proved in separate ways.

This paper consists of two main purposes. The first goal is to construct a theorem for which a number of absolutevalue identities and inequalities can be generated from it. The second is to extend identities and inequalities involving absolute values to an abstract framework of Banach *algebras. In Section 2, after recalling some terminology, we propose an order-preserving linear map from the set of 2by- 2 hermitian matrices to a hermitian Banach $*$-algebra. The image of this map for each 2-by-2 hermitian matrix is in the form involving absolute values. In Section 3, we apply this order preserving to elementary matrix identities and inequalities to get a number of identities and inequalities about absolute values of elements in hermitian Banach *algebras.

\section{An Order-Preserving Linear Map from Matrices to Banach $*$-Algebras}

An element $a$ in a Banach $*$-algebra is called self-adjoint if $a^{*}=a$. An element $a$ which has real spectrum, that is, $\sigma(a) \subseteq \mathbb{R}$, is said to be hermitian. A Banach $*$-algebra is called hermitian if each self-adjoint element is hermitian. The class 
of hermitian Banach *-algebras includes any $C^{*}$-algebra, any group algebra of an abelian group, any group algebra of a compact group, and any measure algebra of discrete group. Throughout this paper, $\mathscr{A}$ denotes a hermitian Banach $*-$ algebra.

Every hermitian Banach *-algebra is equipped with a natural order structure as follows. Given self-adjoint elements $a, b \in \mathscr{A}$, the relation $a \leqslant b$ means that $b-a$ is self-adjoint and $\sigma(b-a) \subseteq[0, \infty)$. Then the relation " $\leqslant$ " forms a partial order on the real vector space of self-adjoint elements in $\mathscr{A}$. The set of $a \in \mathscr{A}$ such that $a \geqslant 0$ forms a positive cone in $\mathscr{A}$ (see [16, Lemma 41.4]), that is, if $a, b \in \mathscr{A}$ are such that $a, b \geqslant 0$, then $\alpha a+\beta b \geqslant 0$ for any $\alpha, \beta \in[0, \infty)$.

The Shirali-Ford Theorem [17, Theorem 1] assures that $a^{*} a \geqslant 0$ for any $a \in \mathscr{A}$. Then the absolute value of $a \in \mathscr{A}$ is defined to be $\left(a^{*} a\right)^{1 / 2}$. By the spectral mapping theorem, $\sigma(|a|) \subseteq[0, \infty)$ and hence $|a| \geqslant 0$ for every $a \in \mathscr{A}$. Note that $|a|=0$ if and only if $a=0$ by [18, Lemma 3].

Denote by $\mathrm{H}_{2}(\mathbb{C})$ the hermitian Banach *-subalgebra of 2-by-2 hermitian matrices of the hermitian Banach *algebra $M_{2}(\mathbb{C})$ of 2 -by-2 complex matrices. The natural order structure on $\mathrm{H}_{2}(\mathbb{C})$ is called the Löwner partial order: the relation $A \leqslant B$ in $H_{2}(\mathbb{C})$ means that $B-A$ is a positive semidefinite matrix, that is, a hermitian matrix with nonnegative eigenvalues.

Theorem 1. Let $a, b \in \mathscr{A}$. The map $\Psi: M_{2}(\mathbb{C}) \rightarrow \mathscr{A}$ given by

$$
\left(\begin{array}{ll}
\alpha & \beta \\
\gamma & \mu
\end{array}\right) \longmapsto \alpha|a|^{2}+\beta a^{*} b+\gamma b^{*} a+\mu|b|^{2},
$$

for $\alpha, \beta, \gamma, \mu \in \mathbb{C}$, is $\mathbb{C}$-linear. The map $\Phi: H_{2}(\mathbb{C}) \rightarrow \mathscr{A}$ given by

$$
\left(\begin{array}{ll}
\alpha & \beta \\
\beta & \gamma
\end{array}\right) \longmapsto \alpha|a|^{2}+\beta a^{*} b+\bar{\beta} b^{*} a+\gamma|b|^{2}
$$

for $\alpha, \gamma \in \mathbb{R}$ and $\beta \in \mathbb{C}$, is $\mathbb{R}$-linear, positive, and orderpreserving. The positivity of $\Phi$ means $A \geqslant 0$ in $\mathrm{H}_{2}(\mathbb{C})$ implies $\Phi(A) \geqslant 0$ in $\mathscr{A}$. The order preserving of $\Phi$ means $A \geqslant B$ in $\mathrm{H}_{2}(\mathbb{C})$ implies $\Phi(A) \geqslant \Phi(B)$ in $\mathscr{A}$.

Proof. The $\mathbb{C}$-linearity of $\Psi$ and the $\mathbb{R}$-linearity of $\Phi$ are clear. For the positivity of $\Phi$, consider $\alpha, \gamma \geqslant 0$ and $\beta \in \mathbb{C}$ such that $\alpha \gamma \geqslant|\beta|^{2}$. If $\beta=0$, we are done. If $\beta \neq 0$, then $\alpha>0$ and $\gamma>0$. Set $\lambda=\alpha \gamma-|\beta|^{2}$. Since $\alpha=\left(\lambda+|\beta|^{2}\right) / \gamma$, it follows that

$$
\begin{aligned}
\alpha|a|^{2} & +\beta a^{*} b+\bar{\beta} b^{*} a+\gamma|b|^{2} \\
& =\frac{\lambda+|\beta|^{2}}{\gamma}|a|^{2}+\beta a^{*} b+\bar{\beta} b^{*} a+\gamma|b|^{2} \\
& =\frac{\lambda}{\gamma}|a|^{2}+\left|\frac{\beta}{\sqrt{\gamma}} a+\sqrt{\gamma} b\right|^{2} \\
& \geqslant 0 .
\end{aligned}
$$

So, $\Phi$ is positive. Now, if $A \leqslant B$ in $H_{2}(\mathbb{C})$, it follows from the linearity and positivity of $\Phi$ that $B-A \geqslant 0$ which implies
$\Phi(B)-\Phi(A)=\Phi(B-A) \geqslant \Phi(0)=0$, that is, $\Phi(A) \leqslant \Phi(B)$.

The most useful form of $\Phi$ in this paper is

$$
\Phi\left(\begin{array}{cc}
|\alpha|^{2} & \bar{\alpha} \beta \\
\alpha \bar{\beta} & |\beta|^{2}
\end{array}\right)=|\alpha a+\beta b|^{2}
$$

The next theorem is very useful to get a necessity and sufficiency condition for the equality case in the later discussions.

Theorem 2. Let $a, b$ be nonzero elements in $\mathscr{A}$. For each $\alpha, \gamma \geqslant$ 0 and $\beta \in \mathbb{C}$ such that $\alpha \gamma \geqslant|\beta|^{2}$, the equation

$$
\alpha|a|^{2}+\beta a^{*} b+\bar{\beta} b^{*} a+\gamma|b|^{2}=0
$$

holds if and only if either $\alpha=\beta=\gamma=0$ or $\alpha \gamma=|\beta|^{2} \neq 0$ and $\alpha a+\beta b=0$. In particular, the restriction of $\Phi$ in the previous theorem to the 2-by-2 positive semidefinite matrices satisfies

$$
\begin{aligned}
\Phi^{-1}(\{0\})= & \left\{\left(\begin{array}{cc}
\alpha & \beta \\
\bar{\beta} & \frac{|\beta|^{2}}{\alpha}
\end{array}\right): \alpha a+\beta b=0, \alpha>0, \beta \in \mathbb{C}\right\} \\
& \cup\left\{\left(\begin{array}{ll}
0 & 0 \\
0 & 0
\end{array}\right)\right\} .
\end{aligned}
$$

Proof. If either $\alpha=\beta=\gamma=0$ or $\alpha \gamma=\beta^{2}$ and $\alpha a+\beta b=0$ holds, then (6) holds. Suppose now that (6) holds for $\alpha, \gamma \geqslant 0$ and $\beta \in \mathbb{C}$ such that $\alpha \gamma \geqslant|\beta|^{2}$. If $\alpha=0$, then $\beta=0$ and $0=\gamma|b|^{2}$, that is, $\gamma=0$. Consider the case $\alpha>0$. If $\gamma=0$, we get $\beta=0$ and then $a=0$, a contradiction. Now for $\gamma>0$, let $\lambda=\alpha \gamma-|\beta|^{2}$. We have $\lambda \geqslant 0$ and $\gamma=\left(\lambda+|\beta|^{2}\right) / \alpha$. Hence

$$
\begin{aligned}
0 & =\alpha|a|^{2}+\beta a^{*} b+\bar{\beta} b^{*} a+\gamma|b|^{2} \\
& =\alpha|a|^{2}+\beta a^{*} b+\bar{\beta} b^{*} a+\frac{\lambda+|\beta|^{2}}{\alpha}|b|^{2} \\
& =\left|\sqrt{\alpha} a+\frac{\beta}{\sqrt{\alpha}} b\right|^{2}+\frac{\lambda}{\alpha}|b|^{2} .
\end{aligned}
$$

This forces $|\sqrt{\alpha} a+(\beta / \sqrt{\alpha}) b|=(\lambda / \alpha)|b|=0$ and hence $\alpha a+$ $\beta b=0$ and $\alpha \gamma=|\beta|^{2}$.

\section{Applications}

The $\mathbb{R}$-linearity and order preserving of the map $\Phi$ and the $\mathbb{C}$-linearity of $\Psi$ are used in order to obtain a number of identities and inequalities from suitable matrix identities and inequalities. We give some applications of Theorem 1 as follows:

(1) parallelogram law and its generalizations,

(2) polarization identity and its generalizations,

(3) Bohr's inequality and its reverse,

(4) generalizations of Bohr's inequality, 
(5) related absolute-value identities and inequalities.

Let $\mathscr{A}$ be a hermitian Banach $*$-algebra.

Corollary 3. Let $a, b \in \mathscr{A}$ and $n \in \mathbb{N}$. If $\omega$ is an nth-root of unity (i.e., $\omega \in \mathbb{C} \backslash\{1\}$ and $\omega^{n}=1$ ), then

$$
|a|^{2}+|b|^{2}=\frac{1}{n} \sum_{k=1}^{n}\left|a+\omega^{k} b\right|^{2}
$$

In particular, the usual parallelogram law holds:

$$
2|a|^{2}+2|b|^{2}=|a-b|^{2}+|a+b|^{2} .
$$

Proof. Since $\omega$ is an $n$ th-root of unity, $\omega^{n-1}+\omega^{n-2}+\cdots+1=0$ and $|\omega|=1$. Then a computation shows that

$$
\begin{aligned}
& \left(\begin{array}{cc}
1 & \omega \\
\bar{\omega} & |\omega|^{2}
\end{array}\right)+\left(\begin{array}{cc}
1 & \omega^{2} \\
\bar{\omega}^{2} & |\omega|^{4}
\end{array}\right)+\cdots+\left(\begin{array}{cc}
1 & \omega^{n} \\
\bar{\omega}^{n} & |\omega|^{2 n}
\end{array}\right) \\
& =\left(\begin{array}{c}
n \\
\bar{\omega}+\bar{\omega}^{2}+\cdots+\bar{\omega}^{n} \\
|\omega|^{2}+|\omega|^{4}+\cdots+|\omega|^{2 n}
\end{array}\right) \\
& =\left(\begin{array}{cc}
n & 0 \\
0 & n
\end{array}\right) .
\end{aligned}
$$

By linearity of $\Psi$ or $\Phi$, we get (9). To get a usual parallelogram law, take $n=2$ and $\omega=-1$.

Corollary 4. Let $a, b \in \mathscr{A}$. Then for any $t \in \mathbb{R} \backslash\{0\}$,

$$
|a+b|^{2}+\frac{1}{t}|t a-b|^{2}=(1+t)|a|^{2}+\left(1+\frac{1}{t}\right)|b|^{2} .
$$

In particular, for $p, q \in \mathbb{R}$ such that $p, q \neq 1, p q>0$ and $1 / p+$ $1 / q=1$,

$$
|a+b|^{2}+\left|\sqrt{\frac{p}{q}} a-\sqrt{\frac{q}{p}} b\right|^{2}=p|a|^{2}+q|b|^{2} .
$$

Proof. Equation (12) is done by applying $\Psi$ or $\Phi$ to a matrix identity

$$
\left(\begin{array}{ll}
1 & 1 \\
1 & 1
\end{array}\right)+\frac{1}{t}\left(\begin{array}{cc}
t^{2} & -t \\
-t & 1
\end{array}\right)=\left(\begin{array}{cc}
1+t & 0 \\
0 & 1+\frac{1}{t}
\end{array}\right)
$$

To get (13), choose $t=p / q>0$ which implies $1+t=p$ and $1+1 / t=q$ from the condition $1 / p+1 / q=1$.

The identities (12) and (13) become the parallelogram law when $t=1$ and $p=q=2$, respectively. The identities (12) and (13) for Hilbert space operators are provided in [11, Theorem 4.1] and [15, Theorem 2], respectively. The identity (13) can be stated equivalently that for any $0 \leqslant \alpha \leqslant 1$,

$$
|\alpha a+(1-\alpha) b|^{2}+\alpha(1-\alpha)|a-b|^{2}=\alpha|a|^{2}+(1-\alpha)|b|^{2} .
$$

Corollary 5. Let $a, b \in \mathscr{A}$ and $n \in \mathbb{N}$. If $\omega$ is an nth-root of unity, then

$$
b^{*} a=\frac{1}{n} \sum_{k=1}^{n} \omega^{k}\left|a+\omega^{k} b\right|^{2} .
$$

In particular, the usual polarization identity holds when we denote $\langle a, b\rangle=b^{*} a\left(\right.$ e.g., in $\left.\mathbb{C}^{n}\right)$ :

$$
\langle a, b\rangle=\frac{1}{4} \sum_{k=1}^{4} i^{k}\left|a+i^{k} b\right|^{2} .
$$

Proof. Since $\omega$ is an $n$ th-root of unity, $\omega^{n-1}+\omega^{n-2}+\cdots+1=0$ and $|\omega|=1$. Note that $\omega^{2}$ is also an $n$ th-root of unity and hence $1+\omega^{2}+\omega^{4}+\cdots+\omega^{2 n-2}=0$. Then a computation shows that

$$
\begin{aligned}
& \omega\left(\begin{array}{cc}
1 & \omega \\
\bar{\omega} & |\omega|^{2}
\end{array}\right)+\omega^{2}\left(\begin{array}{cc}
1 & \omega^{2} \\
\bar{\omega}^{2} & |\omega|^{4}
\end{array}\right)+\cdots+\omega^{n}\left(\begin{array}{cc}
1 & \omega^{n} \\
\bar{\omega}^{n} & |\omega|^{2 n}
\end{array}\right) \\
& =\left(\begin{array}{cc}
\omega+\omega^{2}+\cdots+\omega^{n} & \omega^{2}+\omega^{4}+\cdots+\omega^{2 n} \\
\omega \bar{\omega}+\omega^{2} \bar{\omega}^{2}+\cdots+\omega^{n} \bar{\omega}^{n} & \omega|\omega|^{2}+\omega^{2}|\omega|^{4}+\cdots+\omega^{n}|\omega|^{2 n}
\end{array}\right) \\
& =\left(\begin{array}{cc}
\omega\left(1+\omega+\cdots+\omega^{n-1}\right) & \omega^{2}\left(1+\omega^{2}+\omega^{4}+\cdots+\omega^{2 n-2}\right) \\
|\omega|^{2}+|\omega|^{4}+\cdots+|\omega|^{2 n} & \omega\left(1+\omega+\cdots+\omega^{n-1}\right)
\end{array}\right) \\
& =\left(\begin{array}{ll}
0 & 0 \\
n & 0
\end{array}\right) \text {. }
\end{aligned}
$$

By $\mathbb{C}$-linearity of $\Psi$, we get (16). To get a usual polarization identity, take $n=4$ and $\omega=i$.

Next, consider Bohr's inequality and its extensions. The operator version of Bohr's inequality is first proved in [12, Corallary 1]. The following result gives a generalization of Bohr's inequality and also includes its reverse.

Corollary 6. Let $a, b \in \mathscr{A}$ and $p, q \in \mathbb{R}$ such that $1 / p+1 / q=$ 1.

(i) Bohr's inequality: if $p, q>1$, then

$$
|a+b|^{2} \leqslant p|a|^{2}+q|b|^{2}
$$

with equality if and only if $p a=q b$ (i.e., $b=(p-1) a$ or $a=(q-1) b)$.

(ii) Reverse Bohr's inequality: if $p, q<1$, then

$$
|a+b|^{2} \geqslant p|a|^{2}+q|b|^{2}
$$

with equality if and only if $p a=q b$.

Proof. Let $p, q>1$ be such that $1 / p+1 / q=1$. Then $(p-$ 1) $(q-1)=1$ which implies

$$
\left(\begin{array}{ll}
1 & 1 \\
1 & 1
\end{array}\right) \leqslant\left(\begin{array}{ll}
p & 0 \\
0 & q
\end{array}\right) .
$$

Since $\Phi$ is order preserving, we obtain the Bohr's inequality (19). By Theorem 2, $(p-1)|a|^{2}-a^{*} b-b^{*} a+(q-1)|b|^{2}=0$ if and only if $(p-1) a-b=0$, that is, $b=(p-1) a$ (note that $p, q \neq 1$ ). Part (ii) is similarly proven. 
Corollary 7. Let $a, b \in \mathscr{A}$ and $p, q$ real numbers such that $1 / p+1 / q=1$.

(i) If $p<1$, then

$$
\begin{aligned}
& |a+b|^{2}+|(p-1) a-b|^{2} \geqslant p|a|^{2}+q|b|^{2}, \\
& |a+b|^{2}+|a-(q-1) b|^{2} \geqslant p|a|^{2}+q|b|^{2},
\end{aligned}
$$

with equality if and only if $b=(p-1) a$.

(ii) If $1<p \leqslant 2$, then

$$
\begin{aligned}
& |a+b|^{2}+|(p-1) a-b|^{2} \leqslant p|a|^{2}+q|b|^{2}, \\
& |a+b|^{2}+|a-(q-1) b|^{2} \geqslant p|a|^{2}+q|b|^{2},
\end{aligned}
$$

with equality if and only if $p=q=2$ or $b=(p-1) a$.

(iii) If $p>2$, then

$$
\begin{aligned}
& |a+b|^{2}+|(p-1) a-b|^{2} \geqslant p|a|^{2}+q|b|^{2}, \\
& |a+b|^{2}+|a-(q-1) b|^{2} \leqslant p|a|^{2}+q|b|^{2},
\end{aligned}
$$

with equality if and only if $b=(p-1) a$.

Proof. Assume $1<p \leqslant 2$. The inequality (23) is obtained by applying the order-preserving $\Phi$ to a matrix ordering

$$
\begin{aligned}
\left(\begin{array}{ll}
1 & 1 \\
1 & 1
\end{array}\right)+ & \left(\begin{array}{cc}
(p-1)^{2} & 1-p \\
1-p & 1
\end{array}\right) \\
& =\left(\begin{array}{cc}
p^{2}-2 p+2 & 2-p \\
2-p & 2
\end{array}\right) \leqslant\left(\begin{array}{ll}
p & 0 \\
0 & q
\end{array}\right) .
\end{aligned}
$$

By Theorem 2, $\left(p^{2}-2 p+2-p\right)|a|^{2}+(2-p) a^{*} b+(2-$ $p) b^{*} a+(2-q)|b|^{2}=0$ holds if and only if either $p^{2}-3 p-2=$ $p-2=q-2=0$ or $(p-1)(p-2) a+(2-p) b=0$ and $\left(p^{2}-3 p-2\right)(2-q)=(2-p)^{2}$. The first case is $p=q=2$. The latter case is $b=(p-1) a$ since $\left(p^{2}-3 p-2\right)(2-q)=(2-p)^{2}$ always holds from the fact that $p+q=p q$.

Now, a matrix ordering

$$
\begin{aligned}
\left(\begin{array}{ll}
1 & 1 \\
1 & 1
\end{array}\right)+ & \left(\begin{array}{cc}
1 & 1-q \\
1-q & (q-1)^{2}
\end{array}\right) \\
& =\left(\begin{array}{cc}
2 & 2-q \\
2-q & q^{2}-2 q+2
\end{array}\right) \geqslant\left(\begin{array}{ll}
p & 0 \\
0 & q
\end{array}\right)
\end{aligned}
$$

yields (24) via the map $\Phi$. The proofs of others results are similar to that one.

The analogue results for the case of operators on a Hilbert space are obtained in [10] (cf. Theorem 2, Theorem 1 and Corollary 1 in [10], resp.). The next result generalizes [11, Theorem 3.2].

\section{Corollary 8. Let $a, b \in \mathscr{A}$,}

(a) if $0<t \leqslant 1$, then $|a+b|^{2}+|t a-b|^{2} \leqslant(1+t)|a|^{2}+(1+$ $1 / t)|b|^{2}$ with equality if and only if $t=1$ or $b=t a$, (b) if $t<0$ or $t \geqslant 1$, then $|a+b|^{2}+|t a-b|^{2} \geqslant(1+t)|a|^{2}+$ $(1+1 / t)|b|^{2}$ with equality if and only if $t=1$ or $b=t a$.

Proof. Assume $0<t \leqslant 1$. The order-preserving $\Phi$ brings a matrix inequality

$$
\left(\begin{array}{ll}
1 & 1 \\
1 & 1
\end{array}\right)+\left(\begin{array}{cc}
t^{2} & -t \\
-t & 1
\end{array}\right) \leqslant\left(\begin{array}{cc}
1+t & 0 \\
0 & 1+\frac{1}{t}
\end{array}\right)
$$

to the desired inequality in $\mathscr{A}$. This inequality becomes an equality if and only if

$$
(t-1)\left(t|a|^{2}-a^{*} b-b^{*} a+\frac{1}{t}|b|^{2}\right)=0
$$

which is equivalent to the condition $t=1$ or $t a-b=0$ by Theorem 2. The proof of $(b)$ is similar to that one.

In 2009, generalized Bohr's inequality and its reverse for operators acting on a Hilbert space are done in [9, Theorem 9]. The following results give analogues results in the framework of Banach $*$-algebras.

Corollary 9. Let $a, b \in \mathscr{A}$ and $s, t \in \mathbb{C}, p, q \in \mathbb{R} \backslash\{0\}$ such that $|s|^{2} / p+|t|^{2} / q \leqslant 1$.

(a) Generalized Bohr's inequality: if $|s|^{2} \leqslant p$ and $|t|^{2} \leqslant q$, then

$$
|s a+t b|^{2} \leqslant p|a|^{2}+q|b|^{2} .
$$

(b) Generalized reverse Bohr's inequality: if $|s|^{2} \geqslant p$ and $|t|^{2} \geqslant q$, then

$$
|s a+t b|^{2} \geqslant p|a|^{2}+q|b|^{2} .
$$

In both cases, equality holds if and only if one of the following occurs:

(i) $a=b=0$,

(ii) $a=0, b \neq 0$, and $q=|t|^{2}$,

(iii) $a \neq 0, b=0$, and $p=|s|^{2}$,

(iv) $a \neq 0, b \neq 0,\left(p-|s|^{2}\right) a=\bar{s} t b$, and $|s t|=$ $\sqrt{\left(p-|s|^{2}\right)\left(q-|t|^{2}\right)}$ (equivalently, $a \neq 0, b \neq 0$, $\overline{s t} a=\left(q-|t|^{2}\right) b$ and $|s t|=\sqrt{\left.\left(p-|s|^{2}\right)\left(q-|t|^{2}\right)\right)}$.

Proof. The proofs of (a) and (b) are similar. For (a) assume $|s|^{2} \leqslant p$ and $|t|^{2} \leqslant q$. The conditions $|s|^{2}-p \leqslant 0,|t|^{2}-q \leqslant 0$ and $\left(p-|s|^{2}\right)\left(q-|t|^{2}\right)=|s t|^{2}$ imply

$$
\left(\begin{array}{cc}
|s|^{2} & \bar{s} t \\
s \bar{t} & |t|^{2}
\end{array}\right) \leqslant\left(\begin{array}{cc}
p & 0 \\
0 & q
\end{array}\right) \text {. }
$$

So, by passing through the order-preserving $\Phi$, we obtain (30). When $a \neq 0$ and $b \neq 0$, the equality holds by Theorem 2 if and only if either $p-|s|^{2}=\bar{s} t=q-|t|^{2}=0$ or $\left(p-|s|^{2}\right) a=\bar{s} t b$ and $\left(p-|s|^{2}\right)\left(q-|t|^{2}\right)=|s t|^{2}$. The first case is impossible. The latter case is equivalent to $\bar{s} \bar{t} a=\left(q-|t|^{2}\right) b$ and $|s t|=\sqrt{\left(p-\left.s\right|^{2}\right)\left(q-\left.t\right|^{2}\right)}$. For $a=0$ and $b \neq 0$, it forces $q=|t|^{2}$. For $a \neq 0$ and $b=0$, it forces $p=|s|^{2}$. 
Corollary 10. For any $a, b \in \mathscr{A}$, we have

$$
\pm\left(a^{*} b+b^{*} a\right) \leqslant \frac{1}{2}|a \pm b|^{2} \leqslant|a|^{2}+|b|^{2}
$$

and each equality holds if and only if $a= \pm b$.

Proof. The inequality (33) follows via applying $\Phi$ to a matrix inequality

$$
\left(\begin{array}{cc}
0 & \pm 1 \\
\pm 1 & 0
\end{array}\right) \leqslant \frac{1}{2}\left(\begin{array}{cc}
1 & \pm 1 \\
\pm 1 & 1
\end{array}\right) \leqslant\left(\begin{array}{ll}
1 & 0 \\
0 & 1
\end{array}\right)
$$

Each equality holds if and only if $|a|^{2} \mp a^{*} b \mp b^{*} a+|b|^{2}=0$. By Theorem 2, it holds if and only if $a= \pm b$.

In particular, for any $a, b \in \mathscr{A}$ and $t \in \mathbb{C} \backslash\{0\}$

$$
\pm\left(a^{*} b+b^{*} a\right) \leqslant \frac{1}{2}\left|t a \pm \frac{1}{t} b\right|^{2} \leqslant|t|^{2}|a|^{2}+\frac{1}{|t|^{2}}|b|^{2}
$$

with each equality holds if and only if $b= \pm t^{2} a$. The inequalities of this form for operators appear in [15, Theorem 3].

We finally comment that the order-preserving $\Phi$ can generate many absolute-value identities and inequalities from any 2 -by- 2 matrix inequalities.

\section{References}

[1] H. Bohr, "Zur Theorie der Fastperiodischen Funktionen I," Acta Math, vol. 45, pp. 29-127, 1924.

[2] D. Mitrinovic, Analytic Inequalities, Springer-Verlag, New York, NY, USA, 1970.

[3] D. Mitrinovic, J. Pečarić, and A. Fink, Classical and New Inequalities in Analysis, Kluwer Academic, Dordrecht, The Netherlands, 1993.

[4] P. Vasić and J. Kečkić, "Some inequalities for complex numbers," Mathematica Balkanica, vol. 1, pp. 282-286, 1971.

[5] H. Bergström, "A triangle-inequality for matrices," in Proceedings of the Den 11-te Skandinaviske Matematikerkongress, 1949, pp. 264-267, Trondheim, Oslo, 1952.

[6] S. Abramovich, J. Barić, and J. Pečarić, "A new proof of an inequality of Bohr for Hilbert space operators," Linear Algebra and Its Applications, vol. 430, no. 4, pp. 1432-1435, 2009.

[7] S. Abramovich, J. Barić, and J. Pečarić, "Superquadracity, Bohr's inequality and deviation from a mean value," Australian Journal of Mathematical Analysis and Applications, vol. 7, no. 1, pp. 1-9, 2010.

[8] P. Chansangiam, "Bohr inequalities in $\mathrm{C}^{*}$-algebras," ScienceAsia, vol. 36, no. 4, pp. 326-332, 2010.

[9] P. Chansangiam, P. Hemchote, and P. Pantaragphong, "Generalizations of Bohr inequality for Hilbert space operators," Journal of Mathematical Analysis and Applications, vol. 356, no. 2, pp. 525-536, 2009.

[10] W. S. Cheung and J. Pečarić, "Bohr's inequalities for Hilbert space operators," Journal of Mathematical Analysis and Applications, vol. 323, no. 1, pp. 403-412, 2006.

[11] M. Fujii and H. Zuo, "Matrix order in Bohr inequality for operators," Banach Journal of Mathematical Analysis, vol. 4, no. 1, pp. 21-27, 2010.
[12] O. Hirzallah, "Non-commutative operator Bohr inequality," Journal of Mathematical Analysis and Applications, vol. 282, no. 2, pp. 578-583, 2003.

[13] M. Moslehian, J. Pečarić, and I. Perić, "An operator extension of Bohr's inequality," Bulletin of the Iranian Mathematical Society, vol. 35, pp. 77-84, 2009.

[14] M. Moslehian and R. Rajic, "Generalizations of Bohr's inequality in Hilbert C* -modules," Linear Multilinear Algebra, vol. 58, pp. 323-331, 2010.

[15] F. Zhang, "On the Bohr inequality of operators," Journal of Mathematical Analysis and Applications, vol. 333, no. 2, pp. 1264-1271, 2007.

[16] F. Bonsall and J. Duncan, Complete Normed Algebras, SpringerVerlag, New York, NY, USA, 1973.

[17] S. Shirali and J. Ford, "Symmetry in complex involutory Banach algebras II," Duke Mathematical Journal, vol. 37, no. 2, pp. 275-280, 1970.

[18] B. Sims, "A characterization of a Banach-star-algebras with numerical range," Bulletin of the Australian Mathematical Society, vol. 4, no. 2, pp. 193-200, 1971. 


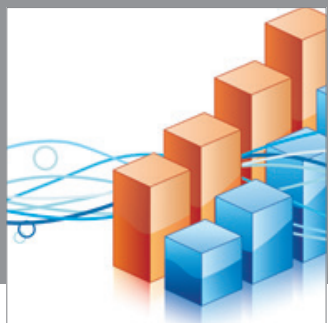

Advances in

Operations Research

mansans

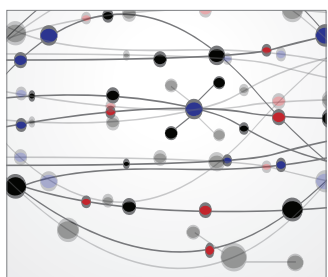

The Scientific World Journal
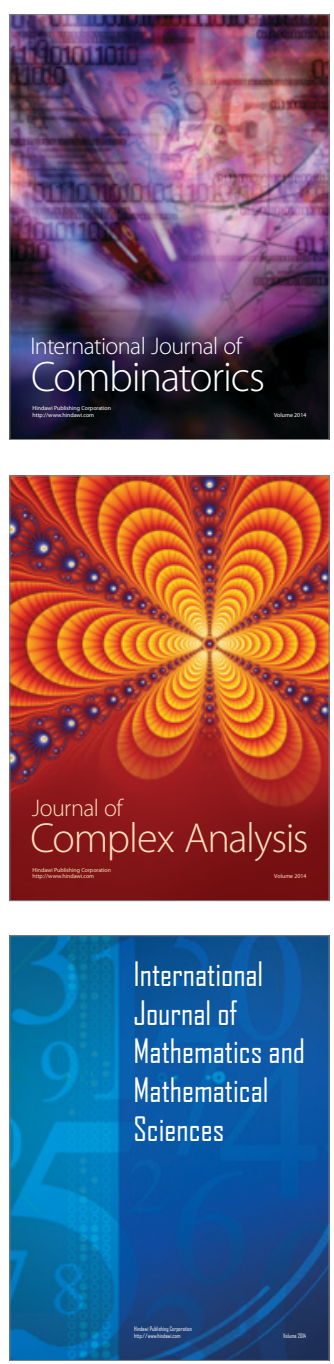
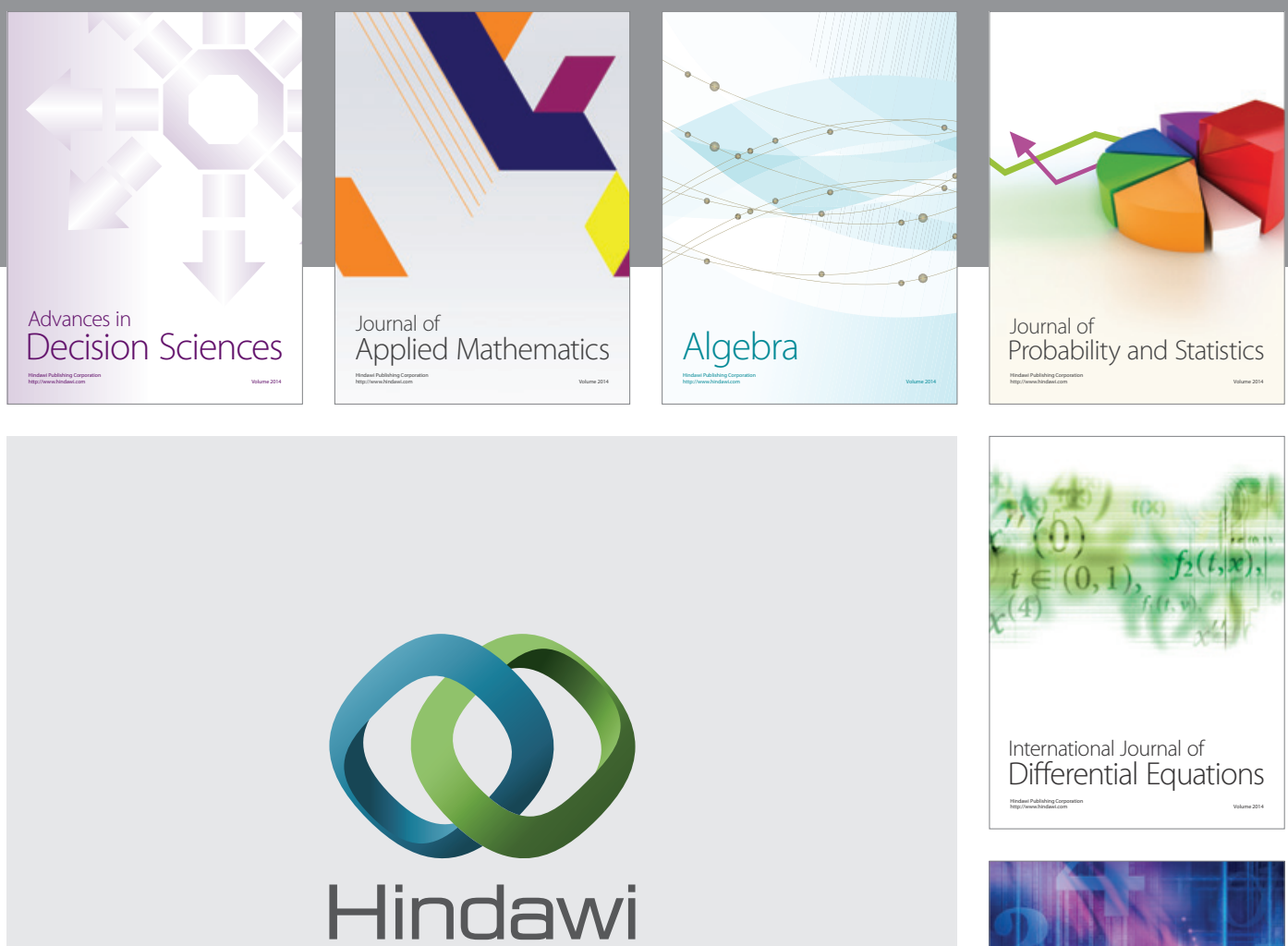

Submit your manuscripts at http://www.hindawi.com
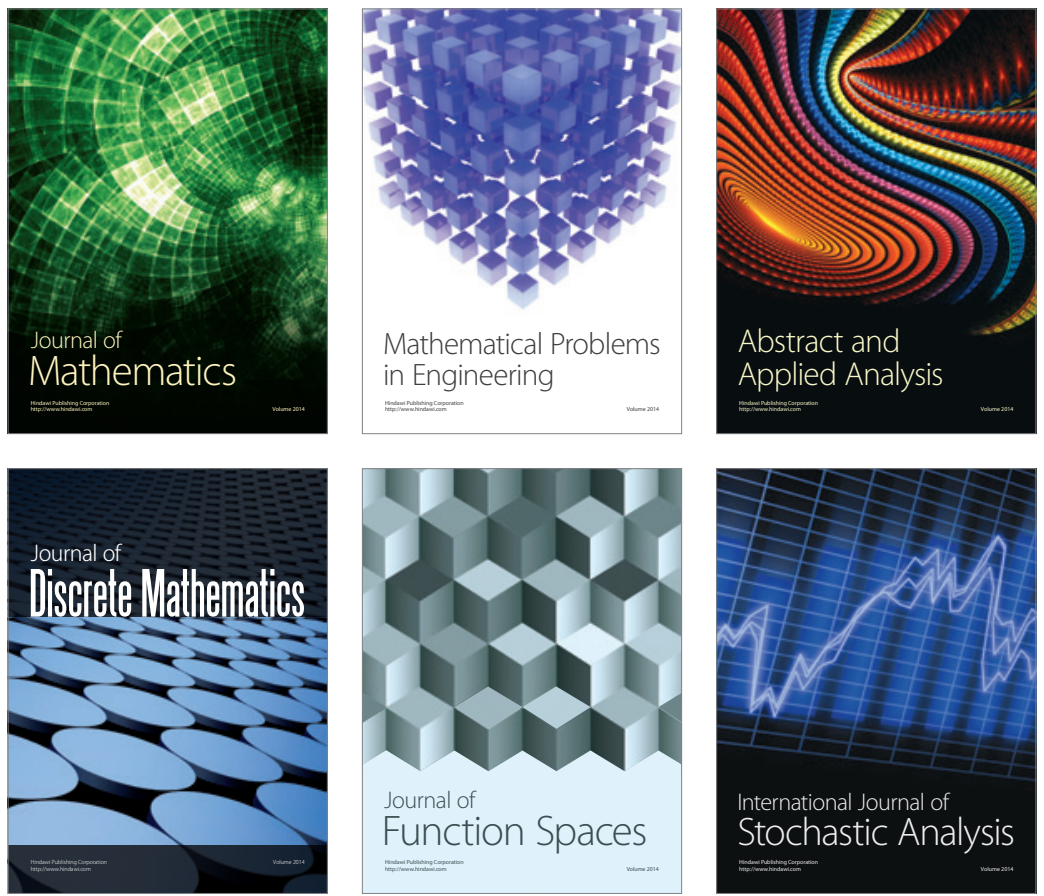

Journal of

Function Spaces

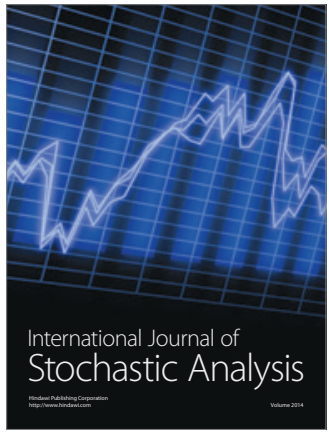

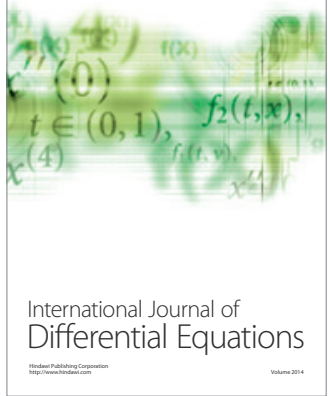
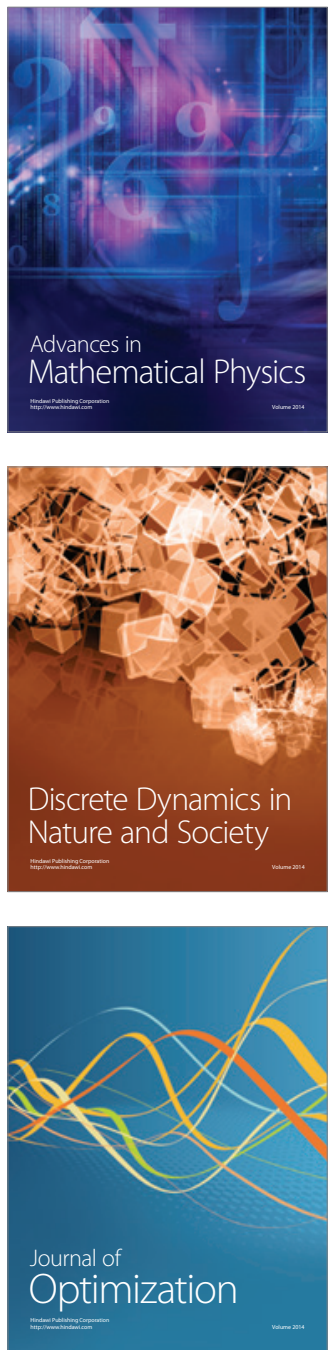